\title{
RUMO À MODELAÇÃO EFICIENTE DE PARÂMETROS GEOTÉCNICOS EM BIM
}

\author{
Mohamad El Sibaii ${ }^{(1)}$, Cátia Marques ${ }^{(2)}$, Luis Bidarra ${ }^{(3)}$, Miguel Azenha ${ }^{(1)}$ \\ (1) ISISE, Dep. Eng. Civil, Escola de Engenharia da Universidade do Minho, Guimarães \\ (2) Instituto Politécnico do Porto, Porto \\ (3) BIM Manager da NRV - Consultores de Engenharia, SA
}

\begin{abstract}
Resumo
A mudança em curso na indústria de Arquitetura, Engenharia e Construção (AEC) caracterizada pelo abandono do uso de métodos tradicionais, rumo ao uso de processos colaborativos baseados no Building Information Modelling (BIM), tem trazido um conjunto de benefícios à construção. Dentro destes benefícios pode destacar-se a maior sustentabilidade, com menos riscos, melhor gestão e melhor desempenho nas construções. A mudança para o BIM teve início no contexto de edifícios, mas rapidamente se estendeu também ao setor de infraestruturas. Neste estudo, pretendeu-se fazer uso de tecnologias de programação em BIM e metodologias de partilha de dados, para melhorar a forma como os dados geotécnicos são usados e preservados. Será dada especial atenção à definição adequada de 'Product Data Templates' (PDT) específicos. As propostas a apresentar compreenderão também cuidados específicos no que diz respeito à interoperabilidade IFC. De forma mais específica, este trabalho tem como objetivo a proposta de metodologia para uso das informações extraídas de sondagens e relatórios geotécnicos em contexto BIM. Nesse sentido, propõe-se um PDT como padrão para armazenamento de dados de sondagens. A metodologia inclui um programa especificamente desenvolvido em plataforma BIM que utilizará dados coerentes com o PDT para gerar representação visual de sondagens e das camadas subterrâneas, anexando automaticamente dados das sondagens modeladas.
\end{abstract}

\section{Introdução}

Até muito recentemente, as marcadas evoluções sentidas em contexto BIM, têm sido limitadas ao contexto da construção de edifícios, com poucos desenvolvimentos abrangentes ao nível do projeto e construção de infraestruturas. Os benefícios da implementação do BIM na infraestrutura estão, no entanto, em franca expansão [1]. Atualmente, recurso a metodologias BIM é já uma prática relativamente generalizada em vários países do mundo, sendo que alguns desses países já tornaram obrigatório o uso do BIM em vários contextos (p.ex., Reino Unido, França, Espanha). Há também várias disposições regulamentares publicadas, que são no entanto maioritariamente destinadas ao contexto de projeto, construção e manutenção de edifícios, sem 
enfoque específico em obras de infraestruturas [2]. No entanto, nos últimos dois anos são várias as evoluções concretas que se pode testemunhar na bibliografia para o contexto das infraestruturas. A investigação nesta área, que parece estar a receber consideravelmente maior atenção atualmente, evidenciando os diversos os benefícios da implementação BIM em infraestruturas [3]. As informações geotécnicas relacionadas com as condições subterrâneas são um dos fatores mais importantes nas fases iniciais de um projeto, que por sua vez, tem grandes impactos no custo e no cronograma do mesmo devido à natureza não uniforme do subsolo [5]. Há um elevado potencial de risco ao encontrar condições inesperadas do solo, estruturas ou infraestruturas de serviços não identificados [6]. A extensão do BIM aos aspetos geotécnicos subterrâneos da infraestrutura é um assunto que tem recebido reduzida atenção na investigação. As condições subterrâneas inesperadas são um dos principais fatores nos atrasos e gastos excessivos dos projetos. Reduzir o risco desses problemas deve ser uma preocupação durante o processo do projeto geotécnico [3]. No presente trabalho é feita uma proposta concreta de 'Product Data Template' (PDT) para sondagens geotécnicas, e efetua-se a sua implementação automática em objetos BIM, com informação alimentada a partir de folha de cálculo (coerente com o PDT). É também criado um automatismo para criação de objetos BIM 3D para representação das camadas geotécnicas (decorrentes da informação das sondagens), contendo a respetiva informação relevante.

\subsection{Relatório geotécnico}

O processo de prospeção geotécnica que é executado através da realização de diferentes sondagens, em diferentes locais para coletar, processar, apresentar e analisar os dados geotécnicos extraídos, envolve uma carga de trabalho pesada. Este processo inclui a recolha de dados geotécnicos (com exemplo ilustrado na figura 1), geológicos, compilação de mapas e análise de dados. O resultado final desse processo é um relatório de investigação.

\begin{tabular}{|c|c|c|c|c|c|c|c|}
\hline \multicolumn{4}{|c|}{ BOREHOLE DRILLING DATA SHEET } & \multicolumn{2}{|l|}{ Borehole No. } & \multicolumn{2}{|l|}{ Page __ of } \\
\hline Project No. & & Project Name & & Site Name & & Supervisor & \\
\hline Start Date & & BH location & & Surface RL & & Groundwater RL & \\
\hline Finsih Date & & Drilling Contractor & & Driller & & Drill Rig & \\
\hline Contractor & & Water Truck & & Water Load Vol. & & Security & \\
\hline Drilling method & From Depth & To Depth & TCR & RQD & Sample no. & SPT N Value & Material Description \\
\hline & & & & & & & \\
\hline & & & & & & & \\
\hline & & & & & & & \\
\hline & & & & & & & \\
\hline & & & & & & & \\
\hline \multicolumn{8}{|l|}{ Time Log } \\
\hline Date & From & To & Activity & Date & From & To & Activity \\
\hline & & & & & & & \\
\hline & & & & & & & \\
\hline \multicolumn{8}{|c|}{ Summary of Quantities } \\
\hline Casing $(\mathrm{m})$ & & SPTs No. & & Core Trays No. & & & \\
\hline Soil Drilling(m) & & Consumed Drill Bits & & Shear Veins No. & & & \\
\hline
\end{tabular}

Figura 1: Exemplo de dados de sondagens [adaptado de 7].

Este relatório é usado pelos engenheiros usualmente para projetos de estruturas subterrâneas como fundações diretas, estacas ou ancoragens, entre outros. Após a fase de projeto e execução da engenharia geotécnica, as outras informações raramente são usadas novamente. 
Normalmente são arquivadas em formato de documento e eventualmente ignoradas mais tarde. Quaisquer intervenções ou projetos futuros no mesmo local por diferentes entidades implicariam muito trabalho para recuperar as informações necessárias, o que resulta em perdas de tempo e dinheiro [8].

\subsubsection{Abordagem tradicional do relatório de investigação geotécnica}

O fluxo tradicional de informações geotécnicas (figura 2) é caracterizado pelo seu processo linear em cascata. Quando um estágio termina, outro começa [9]. Esse processo dificulta a comunicação entre os atores envolvidos, obriga a reinterpretação frequente de informação, acabando por suscitar frequentemente ocorrência de erros e imprecisões. Com a passagem de dados em instantes/contextos específicos em que nem toda a informação é relevante, muitos dados geralmente não são retidos ou reutilizados, o que diminui a eficiência do processo.

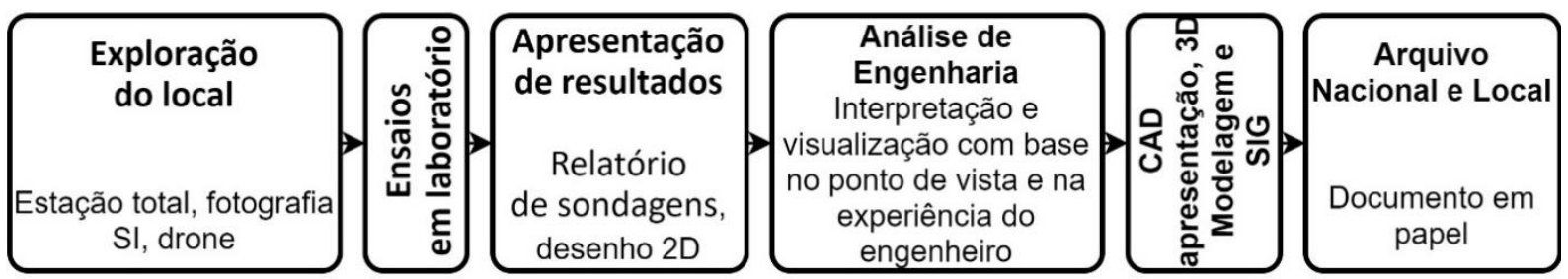

Figura 2: Abordagem Tradicional [adaptado de 9].

\subsubsection{Abordagem BIM ao relatório de investigação geotécnica}

$\mathrm{O}$ processo BIM envolve a gestão de representações digitais das características físicas e funcionais de um projeto [10]. Facilita ainda a partilha de dados entre diferentes equipas de um projeto, para que estas possam trabalhar juntas e de forma colaborativa com mais eficiência [11]. A implementação dessa metodologia na investigação geotécnica de qualquer projeto resultará em maior eficiência e precisão no uso dos dados geotécnicos [12]. Esta provou ser uma metodologia eficiente para economizar tempo e dinheiro em diferentes tipos de projetos em diferentes locais do mundo [13]. O uso de um fluxo de trabalho baseado em BIM, que é um processo circular centrado em dados (figura 3a), melhorará a eficiência no tempo em todas as fases. Como os dados são armazenados numa base de dados BIM, não haverá transmissão de dados no futuro o que diminuirá erros e aumentará a eficiência. À medida que se avança no cronograma temporal do projeto, os dados são acumulados e não são transferidos de uma entidade para outra, tal como na abordagem tradicional. Isto resulta numa maior precisão e melhor preservação dos dados (figura 3b). A padronização do processo BIM, no entanto, ainda é um assunto que merecer a ser explorado com maior detalhe por investigadores desta área no sentido de melhorar os seus resultados [14]. 


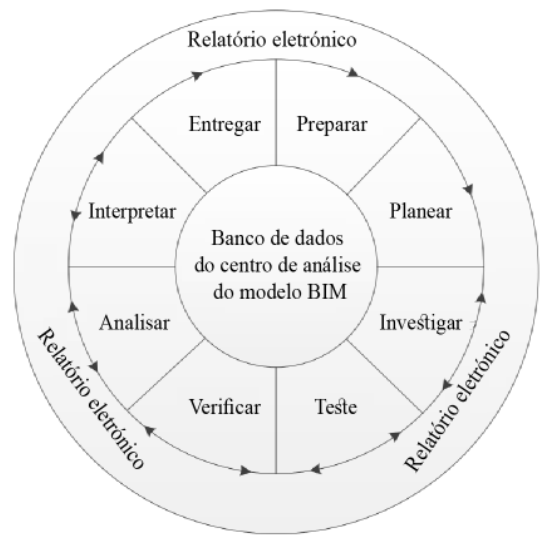

a)

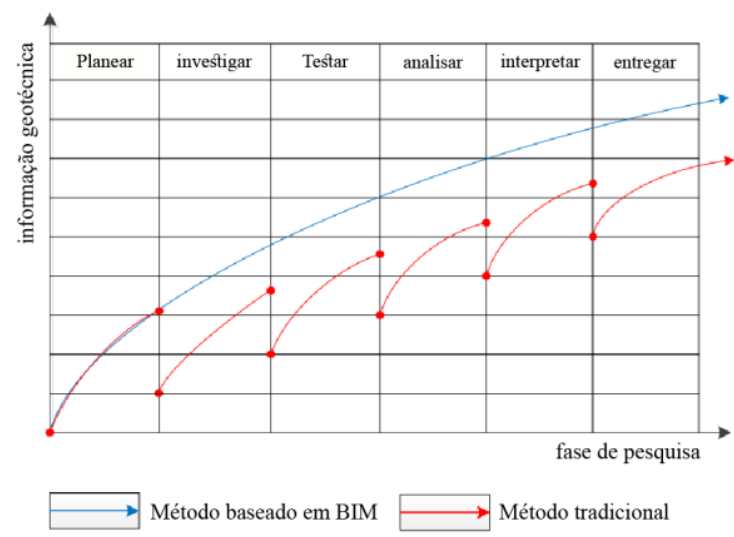

b)

Figura 3: a) O fluxo de trabalho e de dados da investigação geotécnica baseada em BIM; b) a comparação da eficiência de acumulação e uso de informações entre a abordagem baseada em

BIM e a abordagem tradicional nas diferentes fases da investigação geotécnica [14].

\subsection{Ferramentas atuais para modelar camadas subterrâneas}

A Autodesk e a Bentley possuem software específico para a modelação de elementos subterrâneos [15, 16]. Embora estes softwares apresentem grandes benefícios e facilitem o processo de modelação de elementos subterrâneos, não podem verdadeiramente ser considerados como plataformas BIM, em face das suas limitações de interoperabilidade e das lacunas ao nível da capacidade de absorver informação não geométrica. De facto, não é uma tarefa simples nem eficiente quando se alterna entre diferentes softwares de diferentes fornecedores, correndo o risco de perder informações valiosas, especialmente informações geotécnicas relacionadas com as camadas e materiais subterrâneos modelados [17]. As plataformas BIM mais utilizadas no mercado parecem ignorar os aspectos geotécnicos dos modelos, o que causa importantes limitações [18]. A principal dificuldade está na extração e assimilação de dados geotécnicos arquivados entre a plataforma BIM e o fornecedor dos dados propriamente ditos [5,19].

\subsection{Product data template}

Um PDT é um formulário digital para definição das informações mais relevantes a considerar num dado produto para a construção (ou objeto a modelar). Este tem como base as necessidades das diferentes partes interessadas ao longo de todo o projeto, para qualquer produto selecionado [20]. Trata-se de um modelo útil para os engenheiros reduzirem, uniformizarem e otimizarem a entrada de dados no modelo BIM. A adoção de PDT's ainda não se mostrou tão eficiente no atender as necessidades dos ativos do ciclo de vida da infraestrutura, em comparação com o seu estado atual no contexto dos ativos do ciclo de vida dos edifícios [21]. Será dado exemplo de PDT aquando da proposta deste artigo, na secção 2.2.

\subsection{Programação visual}

O recurso a ferramentas de programação visual dentro de software BIM pre-existente (p.ex. Dynamo dentro do Revit) permite aos utilizadores criar programas personalizados, com acesso à base de dados dos modelos, e possibilitar a automatização de tarefas repetitivas, ou extensão de capacidades da plataforma de modelação BIM. Ao usar algoritmos simples para criar 
programas complexos no sentido de resolver diferentes tipos de problemas de engenharia, o potencial é muito grande e depende essencialmente da habilidade e criatividade do programador [22]. O script funciona usando códigos simples, chamados de 'nós', cada um com uma finalidade diferente e com diferentes entradas e saídas. A plataforma é simples, mas fornece todas as ferramentas necessárias para criar a geometria mais complexa e gerir dados. Os desenvolvimentos apresentados neste artigo baseiam-se nesta tipologia de programação.

\subsection{Objectivos e metodologia}

Com base na revisão bibliográfica, não se encontrou nenhum trabalho que abordasse simultaneamente a padronização dos PDTs, a interoperabilidade por meio de IFC e apoio em programação visual para agilizar e melhorar o uso de dados geotécnicos extraídos de sondagens. Neste estudo procura-se colmatar estas lacunas. Procura-se propor forma de melhorar a maneira como o setor de construção interage com as informações geotécnicas, no sentido de garantir o melhor uso dessas informações e fazer a transferência de informações dos relatórios geotécnicos para a plataforma BIM. Pretende-se ainda melhorar e harmonizar a maneira como os dados geotécnicos são usados nos projetos. O caminho para alcançar esse objetivo provém de três estágios principais. Primeiro é a criação do PDT para sondagens, que ajudará na transição de todos os dados dos relatórios geotécnicos para formato digital estruturado. O PDT será criado no formato Excel, que é um formato normalmente acessível a todas as partes interessadas (ou facilmente interoperável com o formato aberto ODS). O segundo é o uso do Dynamo, um software de scripting visual da Autodesk, para projetar uma extensão para a plataforma BIM Revit, que lê a PDT mencionada nas sondagens e traduz automaticamente as informações num modelo 3D de sondagens e camadas de terra com os dados geotécnicos atribuídos anexados. Terceiro, a transformação de todas as informações do modelo no formato IFC para facilitar a interoperabilidade. Os elementos 3D e os dados anexados serão exportados do Revit usando as configurações apropriadas que atendem ao NBS building object standard [24] para exportar elementos IFC.

\section{PDT para Sondagens}

\subsection{Recursos de recolha de dados}

Para que o PDT seja um documento completo que traga benefícios para todas as partes interessadas, é imperativo ter em consideração todas as etapas em que esses dados serão usados. Foram considerados quatro principais recursos para a recolha de dados necessários para o PDT. A primeira fonte é a folha de dados de sondagens, onde todos os dados coletados no local são registados (fig. 1). A segunda fonte é o software de projeto geotécnico, em que a representação das camadas de simulação requer um conjunto de dados específicos (fig. 5). A terceira fonte corresponde às Diretrizes Geotécnicas de Registo de Sondagens do Departamento de Transportes e Estradas Principais do Governo de Queensland [7] assim como as diretrizes de registo adotadas de acordo com as Normas Britânicas [25]. A quarta fonte foi retirada de uma revisão da literatura, onde o tipo de informação geotécnica necessária para projeto geotécnico foi sugeria por especialistas em engenharia geotécnica [6]. Estas continham todos os parâmetros que podem ser extraídos dos dados geotécnicos adicionais a todos os testes laboratoriais e no local de obra realizados assim como os parâmetros relacionados com estes. 


\begin{tabular}{|l|l|}
\hline \multicolumn{1}{|c|}{ Parameters } & \multicolumn{1}{c|}{ Units } \\
\hline Soil Unit Weight & $\mathrm{kN} / \mathrm{m}^{3}$ \\
\hline Young's modulus & $\mathrm{kN} / \mathrm{m}^{2}$ \\
\hline Poisson's ratio & - \\
\hline Cohesion & $\mathrm{kN} / \mathrm{m}^{2}$ \\
\hline Friction angle & Degree \\
\hline Dilatancy angle & Degree \\
\hline
\end{tabular}

Figura 5: Parâmetros necessários para a simulação da mecânica - PLAXIS 2D V20 [26].

\subsection{Proposta de PDT}

Depois de considerar todos os recursos com informação, propõe-se um PDT com total de 104 parâmetros (fig.6), divididos em 5 categorias. A primeira categoria contem informação geral da sondagem, com identificação do projeto, da sua localização e das condições da sondagem. A segunda e terceira categorias contêm os dados sobre a descrição da camada de solo ou rocha. A quarta categoria contém os resultados de classificação, compactação, força e outros testes de laboratório. A última categoria contém os resultados dos testes no local.

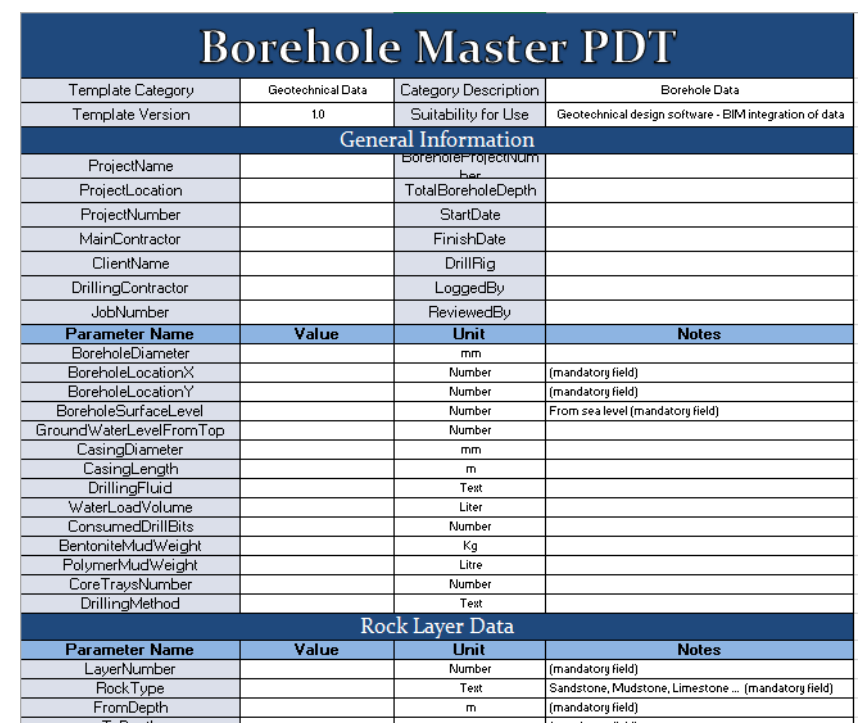

Figura 6: Parte do sondagens PDT.

\section{Modelação Assistida de Dados Geotécnicos}

Usando estratégia baseada em scripting com recurso a programação visual, efetuou-se um pequeno programa de apoio à modelação de sondagens geotécnicas, satisfazendo as informações necessárias do PDT (fig. 8). Para esse propósito, foi criada uma classe de objetos (usando uma família com pontos adaptativos no software Revit) representando a sondagem, contendo todos os parâmetros do PDT como conjuntos de propriedades. Em paralelo foi criada uma folha de Excel contendo todos os elementos do PDT, com formatação adequada para extração de dados preenchidos pelo utilizador possam e exportação direta para programa desenvolvido em Dynamo/Revit para este contexto. Para efeito da modelação 3D das camadas 
subterrâneas, a partir da informação de um conjunto de sondagens, foi criado automatismo adicional (scripting) que lê as informações das sondagens e cria uma forma sólida para representar cada camada respetivamente (fig. 8). Para que o utilizador possa identificar essas camadas mais facilmente, foram adicionados dois parâmetros que identificam o tipo de solo ou rocha que a camada representa.

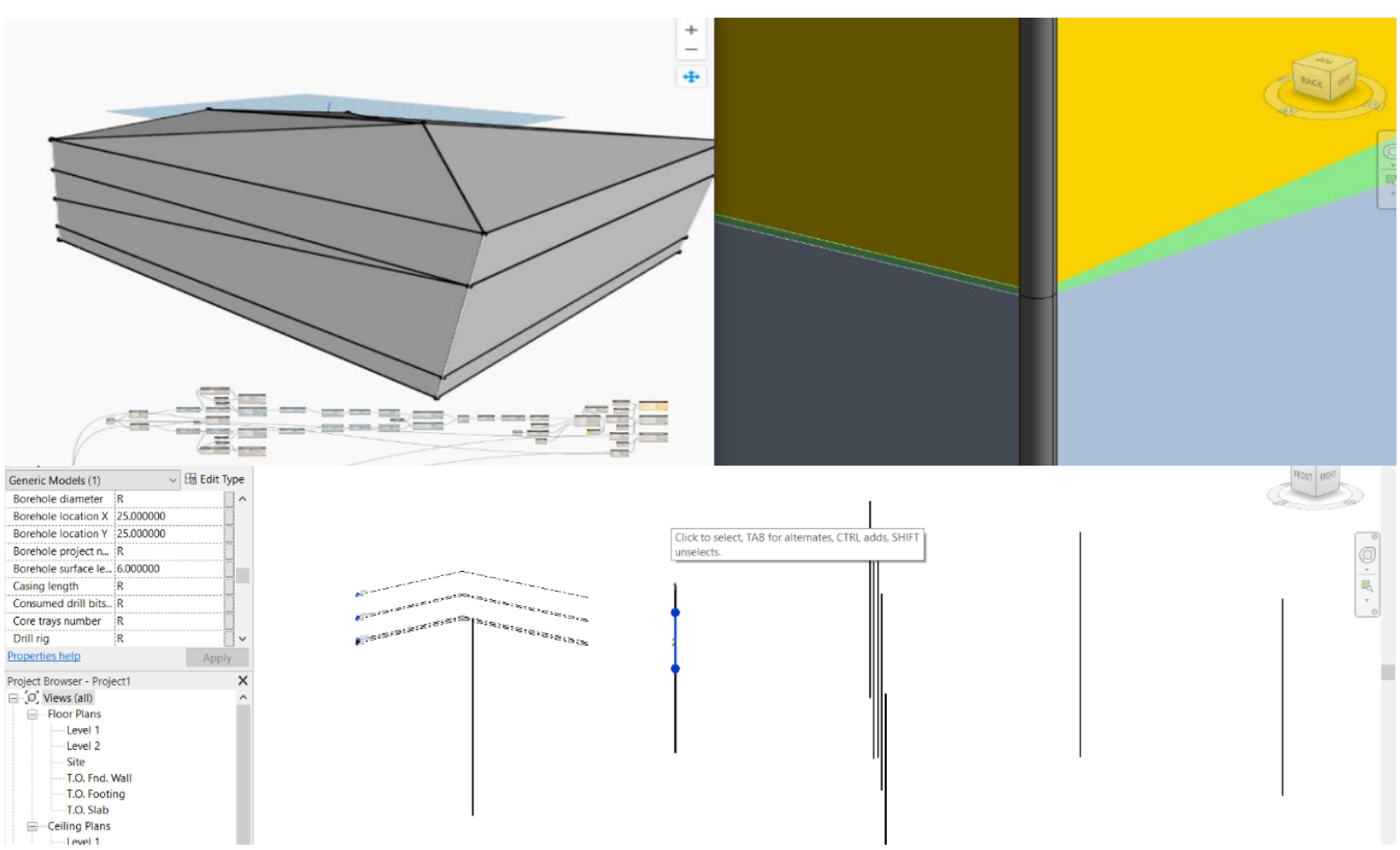

Figura 8: Representação 3D das camadas geotécnicas e sondagens.

\section{Interoperabilidade}

As informações geotécnicas que serão extraídas dos relatórios geotécnicos devem ser preservadas num formato interoperável. Para esse fim, seguiram-se as diretrizes definidas pelo NBS BIM Object Standard [24] para exportar dados no formato IFC4 design transfer view, que afirma que se o elemento não existir na biblioteca IFC, o tipo de objeto "IfcBuildingElementProxy" será usado (fig. 9).

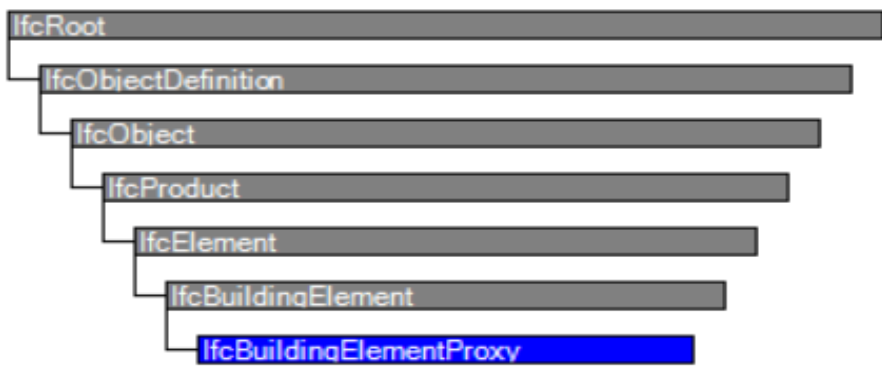

Figura 9: Diagrama de hereditariedade "IfcBuildingElementProxy" [27]. 
De acordo com essas instruções, foi adotada para os elementos de sondagem a designação "IfcBuildingElementProxy", e para a representação de camadas subterrâneas a designação "IfcSite". A implementação de novas capacidades no formato IFC (tal como a iniciativa IFCBridge, por exemplo) permitirá certamente que existam tipologias específicas para alojar este tipo de objetos. Os parâmetros anexados a cada elemento da sondagem são exportados como conjuntos de propriedades IFC e anexados a cada elemento da mesma.

\section{Fluxo de Trabalho e Validação}

Como resultado dos vários desenvolvimentos que foram sendo ilustrados no presente artigo, propõe-se um fluxo de trabalho baseado nas ferramentas desenvolvidas, que leva à preservação de informação geotécnica de forma consistente em modelos BIM, e que se ilustra na fig. 10.

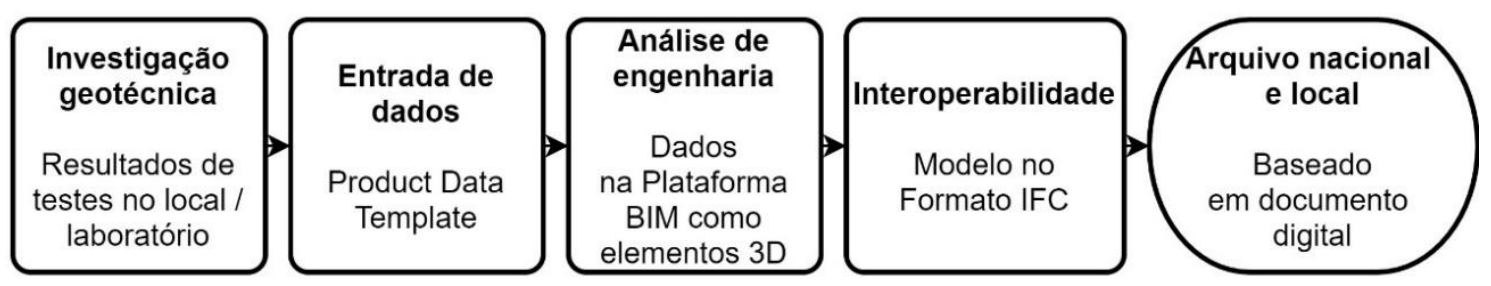

Figura 10: Fluxo de trabalho proposto de dados geotécnicos.

Para validar a metodologia proposta, foram criados dados para 9 diferentes sondagens. Os dados geotécnicos das sondagens foram inseridos na folha de Excel, que inclui todos os parâmetros que constituem o PDT. Em seguida, a família da sondagem foi introduzida no Revit e o código com script foi executado na extensão Dynamo, para implementação dos objetos das sondagens e das camadas subterrâneas (representadas com sólidos 3D). Pode observar-se o resultado final e a exportação do modelo para IFC (na qual se confirmou a passagem de todos os dados) na fig. 11.
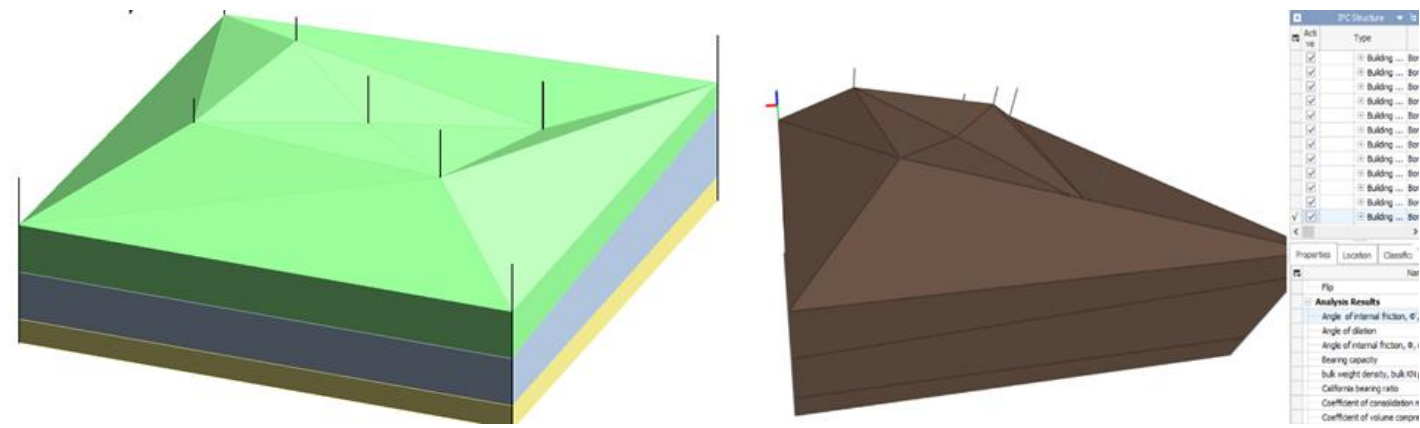

Figura 11: Modelo na plataforma BIM (esquerda) e na plataforma IFC (direita).

O modelo exportado no formato IFC pode ser reimportado para a plataforma BIM Revit. Os dados das sondagens podem subsequentemente exportados no formato de tabela. Esta informacao é importante, uma vez que permite facilmente a exportação de informação para contextos exteriores ao BIM (por exemplo num software geotécnico, ou numa folha de excel proprietária para cálculos internos). 


\section{Conclusões}

As conclusões deste estudo, relacionado com a implementação de informação de sondagens geotécnicas em modelos BIM, centram-se com três aspetos principais. Em primeiro lugar, demonstrou-se a viabilidade de implementar modelos de informação PDT's para este caso em particular, uma vez que não haviam sido encontradas referências a PDT's de sondagens geotécnicas na bibliografia. O segundo diz respeito ao aproveitamento da ferramenta de scripting visual para manipular dados geotécnicos e modelar elementos subterrâneos, que se revelou prática e útil, com melhorarmento da forma como os dados geotécnicos são processados e guardados. O terceiro aspeto relaciona-se com a confirmação de que a interoperabilidade do modelo concluído é possível usando IFC, demonstrando-se a adequada passagem de toda a informação para o formato aberto.

\section{Referencias}

[1] S. Kim and P. Gultekin-Bicer, "An Infrastructure for Geotechnical Building Information Modeling (BIM)," 2018. doi:10.1061/9780784481578.022

[2] A. Bradley, H. Li, R. Lark, and S. Dunn, "BIM for infrastructure: An overall review and constructor perspective," Automation in Construction, vol. 71. Elsevier B.V., pp. 139152, 01-Nov-2016. doi:10.1016/j.autcon.2016.08.019

[3] G. Morin, S. Hassall, and R. Chandler, "Case study - The real life benefits of Geotechnical Building Information Modelling," Inf. Technol. Geo-Engineering. Proc. 2nd Int. Conf. Durham, UK, pp. 95-102, 2014. doi:10.3233/978-1-61499-417-6-95

[4] Scopus 2019. Search results analysis. Retrieved July 13 2019, from https://www.scopus.com/home

[5] J. Zhang, C. Wu, Y. Wang, Y. Ma, Y. Wu, and X. Mao, "The BIM-enabled geotechnical information management of a construction project," Computing, vol. 100, no. 1, pp. 4763, 2018. doi:10.1007/s00607-017-0571-8

[6] L. R. Tawelian and S. B. Mickovski, "The Implementation of Geotechnical Data into the BIM Process," Procedia Eng., vol. 143, no. Ictg, pp. 734-741, 2016. doi:10.1016/j.proeng.2016.06.115

[7] TMR 2019. Geotechnical logging of Queensland Government. Retrieved July 13 2019, from www.tmr.qld.gov.au/business-industry/Technical-standardspublications/Geotechnical-Borehole-Logging

[8] W. Tegtmeier, S. Zlatanova, P. J. M. van Oosterom, and H. R. G. K. Hack, "3D-GEM: Geo-technical extension towards an integrated 3D information model for infrastructural development," Comput. Geosci., vol. 64, pp. 126-135, Mar. 2014. doi:10.1016/j.cageo.2013.11.003

[9] P. Child, C. Grice, and R. Chandler, "The geotechnical data journey-How the way we view data is being transformed," Proc. 2nd Int. Conf. Inf. Technol. Geo-Eng.(ICITG), vol. 3, p. 83, 2014. doi:10.3233/978-1-61499-417-6-83

[10] X. Xu, L. Ma, and L. Ding, “A framework for BIM-enabled life-cycle information management of construction project," Int. J. Adv. Robot. Syst., vol. 11, no. 1, pp. 1-13, 2014. doi:10.5772/58445 
[11] S. Kubota, and I. Mikami, "Data model-centered four-dimensional information management system for road maintenance," Journal of Computing in Civil Engineering, vol. 27, no. 5, pp. 497-510, 2019.

[12] C. Mignard and C. Nicolle, "Merging BIM and GIS using ontologies application to Urban facility management in ACTIVe3D," Comput. Ind., vol. 65, no. 9, pp. 1276-1290, 2014. doi:10.1016/j.compind.2014.07.008

[13] M. Berdigylyjov and H. Popa, "The implementation and role of geotechnical data in BIM process," E3S Web Conf., vol. 85, no. October 2015, pp. 1-8, 2019. doi:10.1051/e3sconf/20198508009

[14] J. Zhang, C. Wu, L. Wang, X. Mao, and Y. Wu, "The Work Flow and Operational Model for Geotechnical Investigation Based on BIM," IEEE Access, vol. 4, pp. 7500-7508, 2016. doi:10.1109/ACCESS.2016.2606158

[15] Bentley 2019. Holebase. Retrieved February 5 2020, from https://www.bentley.com/en/products/product-line/geotechnical-engineeringsoftware/holebase

[16] Autodesk 2019. Civil 3D and Holebase SI. Retrieved February 5 2020, from https://www.autodesk.com/autodesk-university/class/Making-Change-AutoCAD-Civil3D-and-HoleBASE-SI-Geotechnical-BIM-2017

[17] A. Osello, N. Rapetti, and F. Semeraro, "BIM Methodology Approach to Infrastructure Design: Case Study of Paniga Tunnel," IOP Conf. Ser. Mater. Sci. Eng., vol. 245, no. 6, 2017. doi:10.1088/1757-899X/245/6/062052

[18] C. M. Eastman, Y.-S. Jeong, R. Sacks, and I. Kaner, "Exchange Model and Exchange Object Concepts for Implementation of National BIM Standards," J. Comput. Civ. Eng., vol. 24, no. 1, pp. 25-34, 2009. doi:10.1061/(asce)0887-3801(2010)24:1(25)

[19] S. Parry, "Introduction to engineering geology in geotechnical risk management," $Q J$ Eng Geol Hydrogeol, vol. 42, no. 4, pp. 443-444, 2009

[20] CIBSE (2017). CIBSE - Building Information Modelling - BIM. Retrieved July 5, 2019, from www.cibse.org/knowledge/bim-building-information-modelling/product-datatemplates

[21] BuildingSMART (2018). Phil Jackson on behalf of buildingSMART, Infrastructure Asset Managers BIM Requirements, v.1,. Retrieved February 5 2020, from www.buildingsmart.org/wp-content/uploads/2018/01/18-01-09-AM-TR1010.pdf

[22] Autodesk 2020. Dynamo. Retrieved February 5 2020, from https://www.autodesk.com/products/dynamo-studio/overview

[23] Z. Ma and Y. Ren, "Integrated Application of BIM and GIS: An Overview," in Procedia Engineering, 2017, vol. 196, pp. 1072-1079.

[24] NBS 2016. NBS BIM object standard. Retrieved July 14 2019, from https://www.nationalbimlibrary.com/en/nbs-bim-object-standard

[25] RSK 2016, Geotechnical logging manual. Retrieved July 14 2019, from https://www.rsk.co.uk/images/technical-library/brochures/M0348a.pdf

[26] Plaxis (2018). Plaxis 2D Connect edition V20, tutorial manual. Retrieved July 132019 from https://www.plaxis.com/?plaxis_download=2D-1-Tutorial.pdf

[27] BuildingSMART (2019). Retrieved February 5 2020, from https://standards.buildingsmart.org/IFC/RELEASE/IFC4/ADD2_TC1/HTML/schema/ 